\title{
Deep Learning in the Classification of Stage of Liver Fibrosis in Chronic Hepatitis B with Magnetic Resonance ADC Images
}

\author{
Ziquan Zhu $\mathbb{D}^{1},{ }^{1}$ Daoyan $L v \mathbb{D}^{2},{ }^{2}$ Xin Zhang $\mathbb{D}^{,},{ }^{3}$ Shui-Hua Wang $\mathbb{D}^{4},{ }^{4}$ and Guijuan Zhu ${ }^{3}$ \\ ${ }^{1}$ Department of Civil Engineering, University of Florida, Gainesville, FL, USA \\ ${ }^{2}$ Department of Medical Imaging, Huai'an First Hospital, Huai'an, Jiangsu, China \\ ${ }^{3}$ Department of Medical Imaging, The Fourth People's Hospital of Huai'an, Huai'an, Jiangsu, China \\ ${ }^{4}$ School of Architecture Building and Civil Engineering, Loughborough University, Loughborough LE11 3TU, UK \\ Correspondence should be addressed to Xin Zhang; 973306782@qq.com and Shui-Hua Wang; shuihuaw2007@gmail.com
}

Received 12 July 2021; Revised 9 August 2021; Accepted 5 November 2021; Published 22 December 2021

Academic Editor: Ralf Schirrmacher

Copyright $\odot 2021$ Ziquan Zhu et al. This is an open access article distributed under the Creative Commons Attribution License, which permits unrestricted use, distribution, and reproduction in any medium, provided the original work is properly cited.

\begin{abstract}
Liver fibrosis in chronic hepatitis B is the pathological repair response of the liver to chronic injury, which is a key step in the development of various chronic liver diseases to cirrhosis and an important link affecting the prognosis of chronic liver diseases. The further development of liver fibrosis in chronic hepatitis B can lead to the disorder of hepatic lobule structure, nodular regeneration of hepatocytes, formation of a pseudolobular structure, namely, cirrhosis, clinical manifestations of liver dysfunction, and portal hypertension. So far, the diagnosis of liver fibrosis in chronic hepatitis B has been made manually by doctors. However, this is very subjective and boring for doctors. Doctors are likely to be interfered with by external factors, such as fatigue and lack of sleep. This paper proposed a 5-layer deep convolution neural network structure for the automatic classification of liver fibrosis in chronic hepatitis B. In the 5-layer deep convolution neural network structure, there were three convolution layers and two fully connected layers, and each convolution layer was connected with a pooling layer. 123 ADC images were collected, and the following results were obtained: the accuracy, sensitivity, specificity, precision, F1, MCC, and FMI were $88.13 \% \pm 1.47 \%$, $81.45 \% \pm 3.69 \%, 91.12 \% \pm 1.72 \%, 80.49 \% \pm 2.94 \%, 80.90 \% \pm 2.39 \%, 72.36 \% \pm 3.39 \%$, and $80.94 \% \pm 2.37 \%$, respectively.
\end{abstract}

\section{Introduction}

Liver fibrosis in chronic hepatitis B is caused by the excessive accumulation of extracellular matrix proteins, including collagen, that occurs in most types of chronic liver diseases [1]. Liver fibrosis in chronic hepatitis B is the pathological repair response of the liver to chronic injury, which is a key step in the development of various chronic liver diseases to cirrhosis and a vital link affecting the prognosis of chronic liver diseases. The further development of liver fibrosis in chronic hepatitis B can lead to the disorder of hepatic lobule structure, nodular regeneration of hepatocytes, formation of a pseudolobular structure, namely, cirrhosis, clinical manifestations of liver dysfunction, and portal hypertension. Liver fibrosis is histologically reversible, and cirrhosis is difficult to reverse but can be reversed in a few cases.

So far, there are three main methods to diagnose liver fibrosis in chronic hepatitis B. The first is the imaging diagnosis of liver fibrosis in chronic hepatitis B [2]: some signs of liver fibrosis can be found by B-ultrasound, MRI, spiral CT, and color Doppler diagnoses, such as irregular or nodular liver outline, changes of liver parenchymal signal, irregular or nodular shape, an increase of spleen thickness, widening of the portal vein, and spleen vein. However, these influential diagnoses cannot make a clear diagnosis of liver fibrosis and fibrosis degree, so it is often used as the auxiliary diagnosis index in clinic. The second is the pathological diagnosis of liver fibrosis in chronic hepatitis B [3]: clinicalpathological diagnosis of liver fibrosis can not only diagnose liver fibrosis but also understand the degree of development of liver fibrosis and potential liver damage. The third is the diagnosis of liver fibrosis in chronic hepatitis B by serum indicators [4]: serum index is the most widely studied method for the diagnosis of liver fibrosis in chronic hepatitis B. There are hyaluronic acid, III type procollagen, para-type collagen, and laminin in the diagnosis of liver fibrosis in 
chronic hepatitis B. Through the measurement and judgment of its detection value, it is of great value to the diagnosis of liver fibrosis in chronic hepatitis B and the measurement of the degree of liver fiber in chronic hepatitis B. However, the serological indexes are not completely corresponding to the pathological changes of liver fibrosis in chronic hepatitis $B$ at present, so attention should be paid to the differentiation in diagnosis.

The diagnosis of liver fibrosis in chronic hepatitis B is carried out manually by doctors. However, this is very subjective and boring for doctors. Doctors are likely to be interfered with by external factors, such as fatigue, lack of sleep, and so on. With the continuous development of artificial intelligence and computer vision, computer technology has been applied to various fields, such as the analysis of medical images [5]. Subramaniam et al. [6] used CNN to segment and diagnose medical images. Kim et al. [7] used the denoising $\mathrm{CNN}$ (DnCNN) method and trained the network using regular-dose images as ground truth and lowdose images as input. Li et al. [8] proposed an FCN model similar to the u-net structure to regress MR images to CT images. Li et al. [9] proposed a new region-based convolution neural network framework for multitask prediction using an epithelial network header and hierarchical network header. Chen et al. [10] proposed a new CNN architecture, called dense res-induction network (DRINET), to improve the convolution layer to learn the characteristics of medical images. Gu et al. [11] proposed a comprehensive attentionbased CNN (CA-Net) for more accurate and explainable medical image segmentation that is aware of the most important spatial positions, channels, and scales at the same time. Xiao et al. [12] proposed a Multiscale Receptive Field Convolution Neural Network (MRF-CNN) for the segmentation of the liver portal areas in hematoxylin and eosin(H\&E-) stained whole slide images (WSIs). Yu et al. [13] proposed a new liver fibrosis detecting algorithm based on the ultrasound echo amplitude analysis and deep learning to classify normal and fibrosis tissue in computer simulation data. Reddy et al. [14] proposed a novel CAD framework using convolution neural networks and transfer learning (pretrained VGG-16 model).

This paper proposed a 5-layer deep convolution neural network structure for the automatic classification of liver fibrosis in chronic hepatitis B. This paper's main innovations and contributions are as follows:

(i) We proposed an automatic classification method of liver fibrosis in chronic hepatitis B

(ii) We used batch normalization to make the model training more stable and avoid gradient explosion

The remaining structure of this paper is as follows: Section 2 introduces the materials, classification, methods, and CNN structure are given in Section 3, Section 4 mainly discusses the experimental results, and Section 5 is about the conclusion, the shortcomings of this paper, and the future research direction.

\section{Materials}

A total of 123 ADC images were collected from local hospitals with the full knowledge and consent of the patients. All the collected ADC images were divided into stages of $F 0, F 1$, $F 2, F 3$, and $F 4$ according to the internationally used METAVIR method, as shown in Figure 1.

The 123 ADC images of patients with chronic hepatitis B were divided into $F 0-F 4$, among which $F 0$ had 12 patient ADC images, $F 1$ had 26 patient ADC images, $F 2$ had 20 patient ADC images, F3 had 26 patient ADC images, and F4 had 39 patient ADC images, as shown in Table 1. Experienced doctors reconfirmed the identification and classification of all ADC images. This paper used the method binary classification, $F 0$ and $F 1$ as one positive group and $F 2, F 3$, and $F 4$ as one negative group.

\section{Methodology}

This paper mainly proposed a 5-layer deep convolution neural network structure for the automatic classification of liver fibrosis in chronic hepatitis B. Since the neural network was proposed, it has been optimized and deepened by researchers [5]. In the 2012 ImageNet Large-Scale Visual Recognition Challenge (ILSVRC), AlexNet [15] won the championship. Two years later, GoogLeNet [16] won the ILSVRC. In 2014, researchers developed a new deep convolution neural network structure: VGG [17]. The proposed 5-layer deep convolution neural network structure is composed of the input layer, three convolution layers, three pooling layers, two fully connected layers, and the output layer, as shown in Figure 2.

3.1. Convolution. The convolution layer is one of the essential parts of a deep convolution neural network (DCNN). In the DCNN, the convolution layer implements a $2 \mathrm{D}$ convolution for the 3D input and 3D filter, since the channels of both input and filter are the same [18]. The convolution layer has three main characteristics. First, the parameters of the convolution layer are composed of a set of learnable filters. Each filter is small in space (width and height), but the depth is consistent with the input data. Second, it can be seen as an output of a neuron. Neurons only observe a small part of the input data and share parameters with all neurons on the left and right sides of the space. Third, the convolution layer can reduce the number of parameters. Because convolution has the characteristic of "weight sharing," it can reduce the calculation cost and prevent overfitting due to too many parameters.

In the DCNN, the working principle of the convolution layer is the filter scans the input for convolution operation to extract features [19]. Its specific operation is the filter scans the input from left to right and from top to bottom (find the same part as the filter in the input), then multiplies the input and the filter, and then, sums up to get the output. 


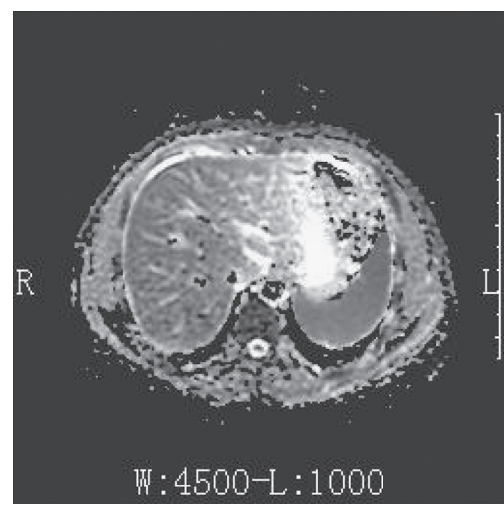

(a)

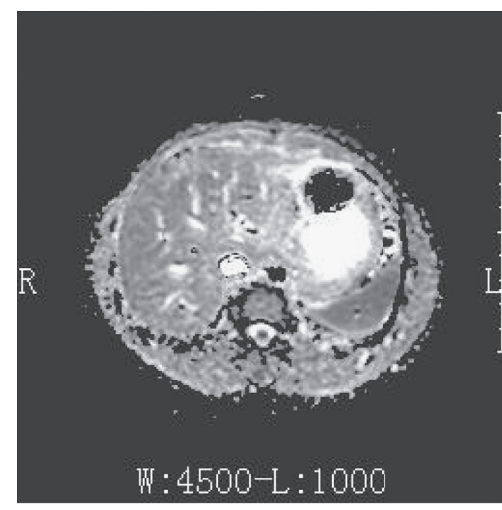

(b)

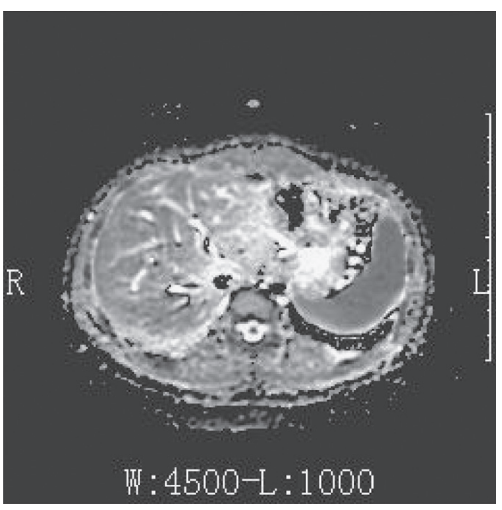

(c)

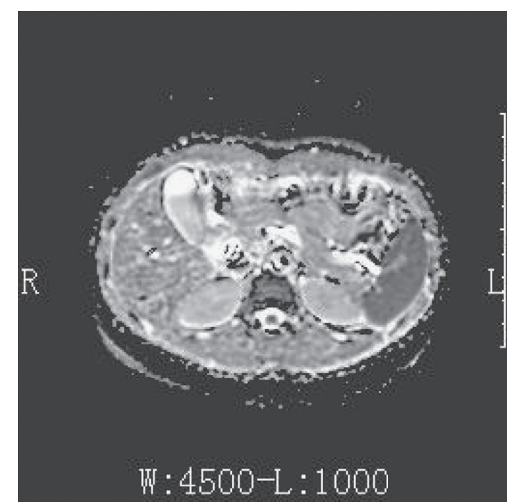

(d)

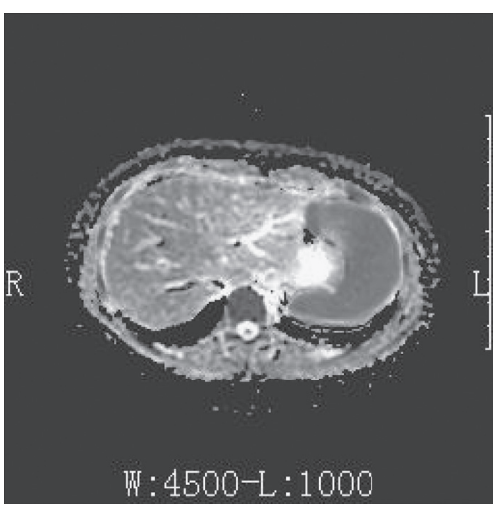

(e)

Figure 1: ADC images. (a) F0. (b) F1. (c) F2. (d) F3. (e) F4.

TABLe 1: Demographics of liver fibrosis in chronic hepatitis B.

\begin{tabular}{llllll}
\hline & $F 0$ & $F 1$ & $F 2$ & $F 3$ & $F 4$ \\
\hline No. of ADC images & 12 & 26 & 20 & 26 & 39 \\
\hline
\end{tabular}

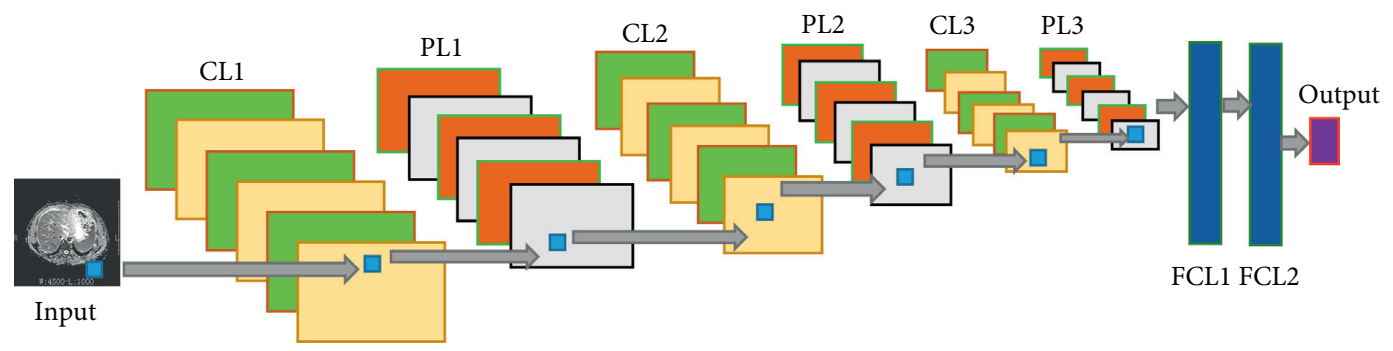

FIGURE 2: The flowchart of our model.

As shown in Figure 3, the input matrix size is $4 \times 4$, the filter matrix size is $3 \times 3$, and the output matrix size is $2 \times 2$. We assume there is an input size with $W_{k} \times H_{k} \times D_{k}$. $W_{k}$ is the width of the input, $H_{k}$ is the height of the input, and $D_{k}$ is the depth of the input. $F_{w} \times F_{h} \times F_{d} . F_{w}$ is the width of the filter, $F_{h}$ is the height of the filter, and $F_{d}$ is the depth of the filter. The number of filters is generally not certain. Researchers usually determine the number of filters by experience. The output is calculated as follows:

$$
\begin{aligned}
W_{k+1} & =\frac{W_{k}-F_{w}+2 B}{Q}+1, \\
H_{k+1} & =\frac{H_{k}-F_{h}+2 B}{Q}+1, \\
D_{k+1} & =M .
\end{aligned}
$$

In the abovementioned formula, the size of the output is $W_{k+1} \times H_{k+1} \times D_{k+1}, B$ represents the padding, $Q$ represents 

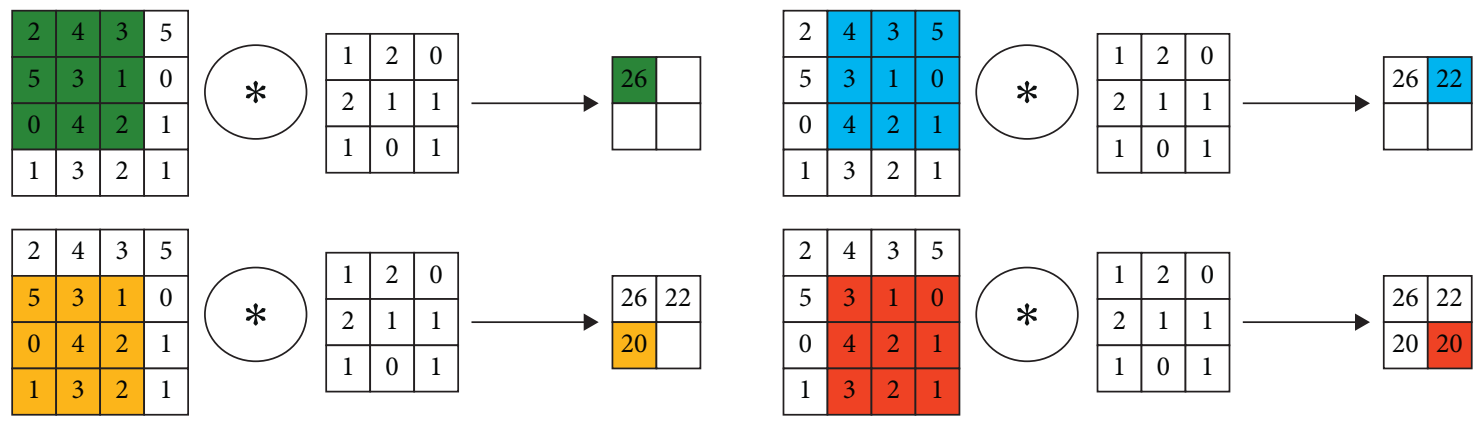

FIgURE 3: Convolution operation.

the stride, and $M$ denotes the number of filters. The flow chart of the convolution layer is shown in Figure 4.

3.2. Pooling. A pooling layer is usually added after one or more convolution layers in the deep convolution neural network. The pooling layer operation does not need a specific kernel. The pooling layer has two advantages: (i) helping to obtain invariance to translation and (ii) reducing the dimension to reduce the amount of calculation [20]. Two pooling layers are commonly used: max pooling and average pooling.

Figure 5(a) shows that this is the max pooling that selects the maximum value within the pool region. The width and height of the pooling layer will be reduced by half with a stride of 2 . The output size of the pooling operation is a matrix with $2 \times 2$, while the input size is a matrix with $4 \times 4$. Pooling operation makes the dimension of input size greatly smaller.

As shown in Figure 5(b), the working principle of average pooling is similar to that of maximum pooling, but the average value replaces the maximum value.

Suppose a rectangular region $R_{i j}$ is given, where $i$ is the number of rows and $j$ is the number of columns. The max pooling formula is as follows:

$$
m_{i j}=\max x_{p q}, \quad p=1, \ldots, i \text { and } q=1, \ldots, j,
$$

where $m_{i j}$ represents the output value of the rectangular region $R_{i j}$ by the operation of the max pooling and $x_{p q}$ represents the element at $(p, q)$ in the rectangular region $R_{i j}$.

The average pooling (AP) formula is as follows:

$$
a_{i j}=\frac{1}{\left|R_{i j}\right|} \sum x_{p q}, \quad p=1, \ldots, i \text { and } q=1, \ldots, j \text {, }
$$

where $a_{i j}$ represents the output value of the rectangular region $R_{i j}$ by the operation of the average pooling, $x_{p q}$ represents the element at $(p, q)$ in the rectangular region $R_{i j}$, and $\left|R_{i j}\right|$ represents the number of elements in the rectangular region $R_{i j}$.

3.3. Batch Normalization. For the general neural training model, data standardization has been able to complete the training. However, with the increase of the number of layers and the updating of the parameters in each layer, the results closer to the output layer would change greatly. It is challenging to train deep convolution neural networks effectively [21]. During the model training, batch normalization (BN) uses the mean and standard deviation of small batch to adjust the intermediate output of the neural network continuously [22] so that the value of the intermediate output of the whole neural network in each layer is more stable.

Firstly, the batch $B$ is set as

$$
B=\left\{x_{1}, x_{2}, \ldots, x_{n}\right\},
$$

where $n$ is the number of elements in the batch $B$.

Then, the mean value of the batch $B$ is calculated as follows:

$$
\mu_{B} \leftarrow \frac{1}{n} \sum_{i=1}^{n} x_{i}
$$

The variance is calculated as follows:

$$
\sigma_{B}^{2} \leftarrow \frac{1}{n} \sum_{i=1}^{n}\left(x_{i}-\mu_{B}\right)^{2} .
$$

After calculating the mean and variance, the standardized calculation is carried out:

$$
z_{i} \leftarrow \frac{x_{i}-\mu_{B}}{\sqrt{\sigma_{B}^{2}+\epsilon}}
$$

$\epsilon \in(\epsilon>0)$ is a very small constant, which guarantees that the denominator is greater than 0 . Based on the abovementioned standardization, two model parameters (scale parameter $\gamma$ and shift parameter $\beta$ ) are introduced into the batch normalization layer to get the output:

$$
y_{i} \longleftarrow \gamma z_{i}+\beta
$$

3.4. Rectified Linear Unit. The operation of the activation function is to activate some neurons in the neural network and transmit the activation information to the next layer of the neural network. The neural network can solve nonlinear problems because the activation function adds nonlinear factors, which makes up for the expressive power of the linear model and preserves and maps the "characteristics of activated neurons" to the next layer through functions. In this paper, we used the ReLU function, as shown in Figure 6. 


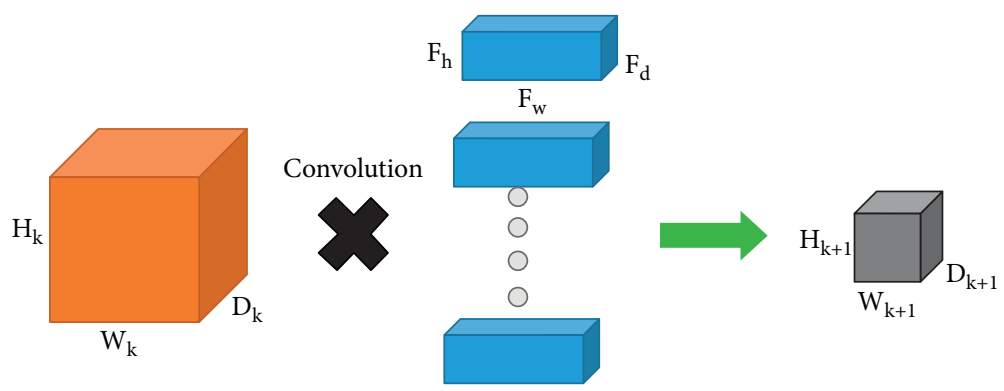

FIGURE 4: Convolution layer flow chart.

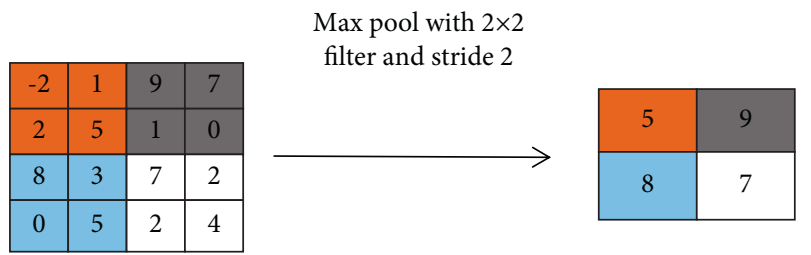

(a)

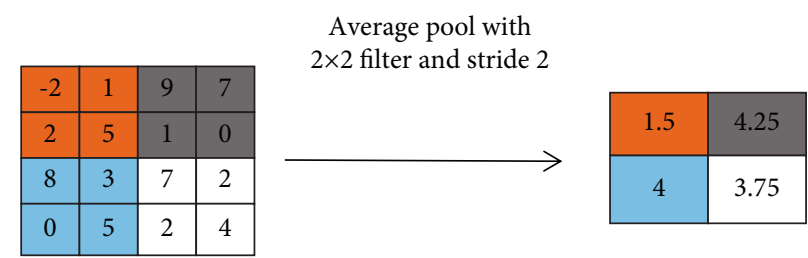

(b)

Figure 5: Pooling layer. (a) Max pooling. (b) Average pooling.

It can be seen from Figure 6 that ReLU is hard saturated when $y \leq 0$. When $y>0$, the ReLU can keep the gradient unchanged. The formula is as follows:

$$
\operatorname{ReLU}(y)= \begin{cases}y, & \text { if } y>0 \\ 0, & \text { if } y \leq 0\end{cases}
$$

From Figure 6 and formula (9), we can see that the ReLU activation function has several advantages: (i) in the case of backpropagation, ReLU can avoid the problem of gradient vanishing, (ii) ReLU makes the output of some neurons zero, which leads to the sparsity of the network, reduces the interdependence of parameters, and alleviates the overfitting problem, and (iii) compared with other activation functions, such as tanh and sigmoid, ReLU calculation is very simple.

3.5. Structure of DCNN. In this paper, the proposed 5-layer deep convolution neural network structure was composed of three convolution layers and two fully connected layers, as shown in Table 2. Each convolution layer was connected with a pooling layer. Each convolution layer had a different number of convolution kernels. The first convolution layer had 32 convolution kernels, the second had 64 convolution kernels, and the third had 96 convolution kernels. The convolution kernel of each convolution layer was $3 \times 3$. After three times convolution and pooling calculations, the parameter was 24576 . The parameters of the first fully connected layer to the second layer were $24576 \times 300$. The output of the second fully connected layer was $300 \times 2$. The flow chart of the DCNN structure is as shown in Figure 7.

3.6. Measures. We use 10-fold cross validation to evaluate our model. We set $w \in 1, \ldots, 10$, and the confusion matrix is set as

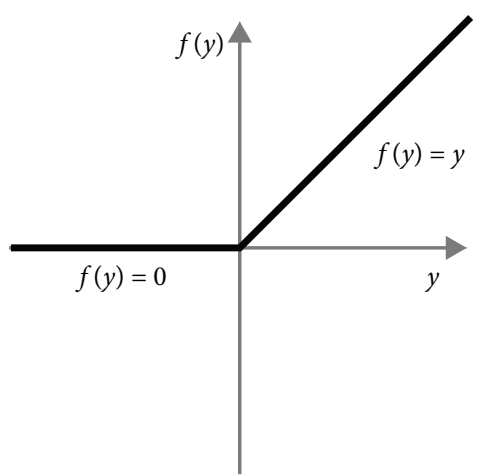

FIGURE 6: ReLU.

TABLE 2: DCNN structure.

\begin{tabular}{lcc}
\hline Layer & Size & Parameters \\
\hline Input & $256 \times 256$ & \\
Conv. layer 1 & $64 \times 64 \times 32$ & $323 \times 3$, pooling size $=2$ \\
Conv. layer 2 & $32 \times 32 \times 64$ & $643 \times 3$, pooling size $=2$ \\
Conv. layer 3 & $16 \times 16 \times 96$ & $963 \times 3$, pooling size $=2$ \\
FCL1 & $300 \times 1$ & $24576 \times 300$ \\
FCL2 & $2 \times 1$ & $300 \times 2$ \\
\hline
\end{tabular}

$$
M(w)=\left[\begin{array}{cc}
\mathrm{TP}(w) & \mathrm{FN}(w) \\
\mathrm{FP}(w) & \mathrm{TN}(w)
\end{array}\right], \quad w \in 1, \ldots, 10
$$

where $M(w)$ is the confusion matrix of the $w$-th run, $\operatorname{TP}(w)$ represents the true positive of the $w$-th run, $\mathrm{FN}(w)$ represents the false negative of the $w$-th run, $\operatorname{FP}(w)$ represents the false positive of the $w$-th run, and $\mathrm{TN}(w)$ represents the true negative of the $w$-th run.

We can define measures as 


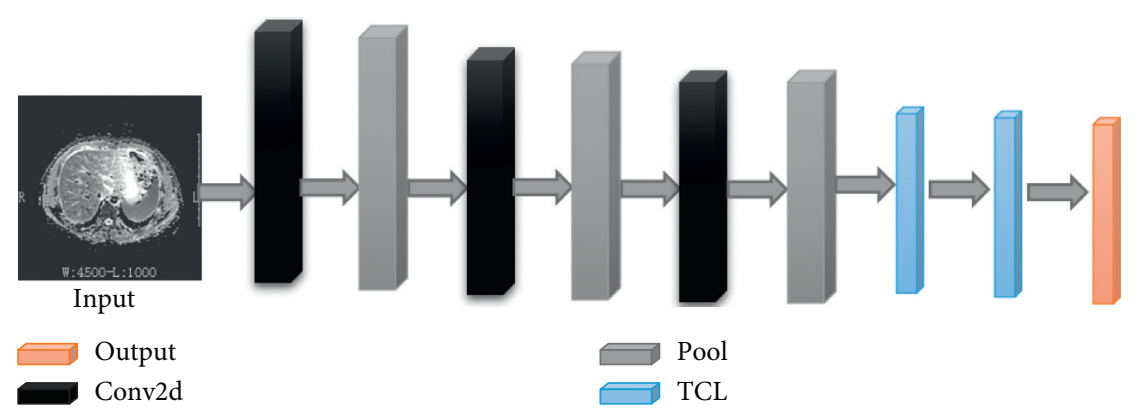

Figure 7: DCNN flow chart.

$$
\begin{aligned}
& t_{1}(w)=\frac{\mathrm{TP}(w)+\mathrm{TN}(w)}{\mathrm{TP}(w)+\mathrm{FP}(w)+\mathrm{TN}(w)+\mathrm{FN}(w)}, \quad w \in 1, \ldots, 10 \\
& t_{2}(w)=\frac{\mathrm{TP}(w)}{\operatorname{TP}(w)+\mathrm{FP}(w)}, \quad w \in 1, \ldots, 10 \\
& t_{3}(w)=\frac{\mathrm{TN}(w)}{\operatorname{FP}(w)+\mathrm{TN}(w)}, \quad w \in 1, \ldots, 10 \\
& t_{4}(w)=\frac{\mathrm{TP}(w)}{\operatorname{TP}(w)+\mathrm{FN}(w)}, \quad w \in 1, \ldots, 10 \\
& t_{5}(w)=\frac{2 \mathrm{TP}(w)}{2 \mathrm{TP}(w)+\mathrm{FP}(w)+\mathrm{FN}(w)}, \quad w \in 1, \ldots, 10 \\
& t_{6}(w)=\frac{\operatorname{TP}(w) \times \mathrm{TN}(w)-\mathrm{FP}(w) \times \mathrm{FN}(w)}{\sqrt{(\mathrm{TP}(w)+\mathrm{FP}(w)) \times(\mathrm{TP}(w)+\mathrm{FN}(w)) \times(\mathrm{TN}(w)+\mathrm{FP}(w)) \times(\mathrm{TN}(w)+\mathrm{FN}(w))}}, \quad w \in 1, \ldots, 10 \\
& t_{7}(w)=\frac{\mathrm{TP}(w)}{\sqrt{(\mathrm{TP}(w)+\mathrm{FP}(w)) \times(\mathrm{TP}(w)+\mathrm{FN}(w))}}, \quad w \in 1, \ldots, 10
\end{aligned}
$$

where $t_{1}$ means accuracy, $t_{2}$ means precision, $t_{3}$ means specificity, $t_{4}$ means sensitivity, $t_{5}$ means $F 1, t_{6}$ means MCC, and $t_{7}$ means FMI.

We calculate the mean $\mu$ and standard deviation SD of all the measures $(h \in 1, \ldots, 7)$ :

$$
\begin{aligned}
\mu\left(t_{h}\right) & =\frac{1}{10} \sum_{w=1}^{10} t_{h}(w), \\
\mathrm{SD}\left(t_{h}\right) & =\sqrt{\frac{1}{10-1} \sum_{w=1}^{10}\left[t_{h}(w)-\mu\left(t_{h}\right)\right]^{2} .}
\end{aligned}
$$

ROC (receiver operating characteristic) curve: each point on the ROC curve reflects the sensitivity to the same signal stimulation, as shown in Figure 8.

AUC (area under the curve): the area under the ROC curve, between 0.1 and 1. AUC as a numerical value can directly evaluate the quality of the classifier. AUC value is a probability value. The larger the AUC value, the better the current classification algorithm can classify.
3.7. Statistics. 10 -fold cross validation is used to evaluate the proposed structure, as shown in Figure 9. The dataset is divided into ten parts, nine of which are used as training data and one as test data. Each test gets the corresponding correct rate (or error rate). Ten groups of data are obtained from 10-fold cross validation. The average values of ten groups are used as the evaluation values. To reduce the contingency caused by a single division of training set and test set, the existing dataset is used to partition multiple times. Cross validation is used to reduce contingency and improve generalization ability.

\section{Experiments}

The results of 10-fold cross validation are given in Table 3 . Among the ten groups of data, the sensitivity of the ninth group was the highest (86.84), and the sensitivity of the tenth group was the lowest (73.68). The specificity of the tenth group was the highest (95.33), and the specificity of the first group was the lowest (88.24). The highest precision was 83.58 in the tenth group, and the lowest was 75.00 in the first group. The highest accuracy was (90.65) in the ninth group, and the lowest (85.37) 


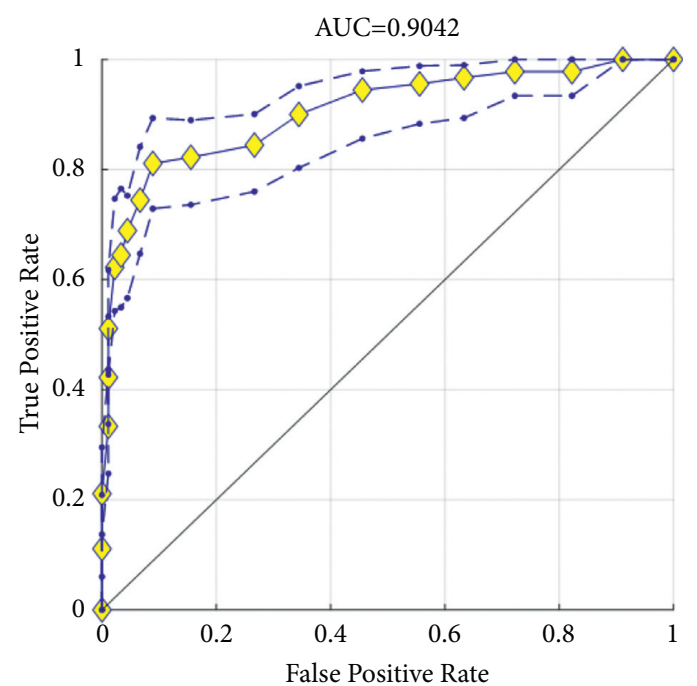

FIgURE 8: ROC curve.

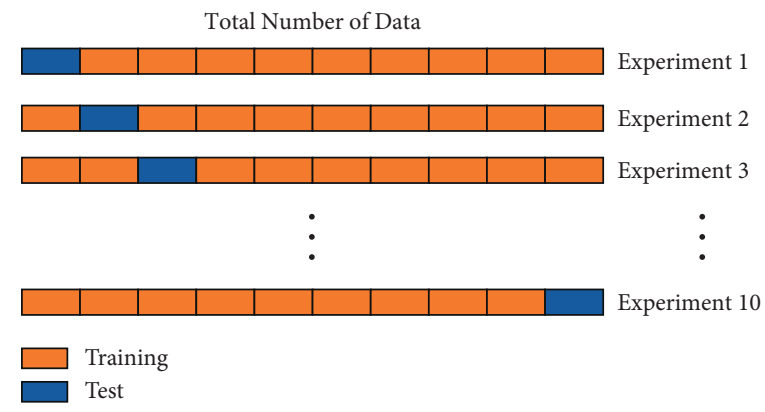

FIgURE 9: An example of 10-fold cross validation.

TABLE 3: Experimental results.

\begin{tabular}{lccccccc}
\hline Run & Sen & Spc & Prc & Acc & $F 1$ & MCC & FMI \\
\hline 1 & 78.95 & 88.24 & 75.00 & 85.37 & 76.92 & 66.27 & 73.16 \\
2 & 80.26 & 92.35 & 82.43 & 88.62 & 81.33 & 81.34 \\
3 & 81.58 & 91.76 & 81.58 & 88.62 & 81.58 & 73.34 \\
4 & 81.58 & 88.82 & 76.54 & 86.59 & 78.98 & 69.22 & 71.58 \\
5 & 84.21 & 89.41 & 78.05 & 87.80 & 81.01 & 72.16 \\
6 & 80.26 & 91.76 & 81.33 & 88.21 & 80.79 & 72.29 \\
7 & 81.58 & 91.76 & 81.58 & 88.62 & 81.58 & 73.34 & 80.80 \\
8 & 85.53 & 91.18 & 81.25 & 89.43 & 83.33 & 75.66 & 81.58 \\
9 & 86.84 & 92.35 & 83.54 & 90.65 & 85.16 & 78.37 & 83.36 \\
10 & 73.68 & 95.33 & 83.58 & 87.40 & 78.32 & 69.76 \\
MSD & $81.45 \pm 3.69$ & $91.12 \pm 1.72$ & $80.49 \pm 2.94$ & $88.13 \pm 1.47$ & $80.90 \pm 2.39$ & $72.36 \pm 3.39$ & $80.94 \pm 2.37$ \\
\hline
\end{tabular}

accuracy was in the first group. The maximum value of $F 1$ was 85.16 in the ninth group, and the minimum value was 92.98 in the first group. The maximum MCC value was 78.37 in the ninth group, and the minimum was 66.27 in the first group. The maximum FMI value was 85.18 in the ninth group, and the minimum was 76.95 in the first group.

It can be concluded from the table that the ninth group of data was the best among the ten groups. The first group of data was the worst, and each data in the first group was the lowest in ten groups.
We introduced the AUC curve and its implications in Section 3.6. Generally speaking, when $\mathrm{AUC}=1$, it is a perfect classifier; when AUC is $[0.85,0.95]$, the classifier is very good; when AUC is [0.7, 0.85], the classifier is general; when AUC is $[0.5,0.7]$, the classifier is bad, when AUC is 0.5 , the model has no predictive value; and when AUC $<0.5$, the classifier is worse than a random guess. As shown in Figure 8 , horizontal axis: false positive rate (FPR) and vertical axis: true positive rate (TPR). The AUC value is 0.9042 , which proves that our method is highly accurate. 


\section{Conclusions}

This paper proposed a 5-layer deep convolution neural network structure for the automatic classification of liver fibrosis in chronic hepatitis B. We used 10-fold cross validation to evaluate the proposed 5-layer deep convolution neural network structure and obtained the following results: the accuracy, sensitivity, specificity, precision, F1, MCC, and FMI were $88.13 \% \pm 1.47 \% 81.45 \% \pm 3.69 \%, 91.12 \% \pm 1.72 \%$, $80.49 \% \pm 2.94 \%, \quad 80.90 \% \pm 2.39 \%, \quad 72.36 \% \pm 3.39 \%$, and $80.94 \% \pm 2.37 \%$, respectively.

The limitations of this study: (i) the training dataset is relatively small. As the number of cases increases and the number of training increases, the system's performance will achieve higher accuracy; (ii) the data collected in this study came from the same hospital. We plan to collect other MRI exams from different centers to evaluate the efficiency of the testing; (iii) we did not compare DCNN structures with the different number of convolution and fully connected layers; and (iv) we did not compare with other approaches.

In the future study, (i) we will collect more data from different sources; (ii) in the next paper, we will do a comparative test on the DCNN structure to get the best DCNN structure.

\section{Data Availability}

The research data used to support the findings of this study are restricted to protect patient privacy.

\section{Disclosure}

Ziquan Zhu and Daoyan Lv should be regarded as the cofirst authors.

\section{Conflicts of Interest}

There are no conflicts of interest regarding the publication of this article.

\section{Authors' Contributions}

Ziquan Zhu and Daoyan Lv contributed equally.

\section{Acknowledgments}

This work was supported by the China Public Health Alliance Project (GWLM202016); "Six one projects" for HighLevel Health Talents in Jiangsu Province in 2020 (LGY2020059).

\section{References}

[1] R. Bataller and D. A. Brenner, "Liver fibrosis," Journal of Clinical Investigation, vol. 115, no. 2, pp. 209-218, 2005.

[2] J. Y.-K. Cheng and G. L.-H. Wong, "Advances in the diagnosis and treatment of liver fibrosis," Hepatoma Research, vol. 3, no. 8, 2017.

[3] Y. Lurie, M. Webb, R. Cytter-Kuint, S. Shteingart, and G. Z. Lederkremer, "Non-invasive diagnosis of liver fibrosis and cirrhosis," World Journal of Gastroenterology, vol. 21, no. 41, pp. 11567-11583, 2015.

[4] D. Laharie, F. Zerbib, X. Adhoute et al., "Diagnosis of liver fibrosis by transient elastography (fibroscan) and non-invasive methods in crohn's disease patients treated with methotrexate," Alimentary Pharmacology and Therapeutics, vol. 23, no. 11, pp. 1621-1628, 2006.

[5] Z. Zhu, "Alcoholism via 6-layer customized deep convolution neural network," STEMedicine, vol. 2, no. 7, p. e93, 2021.

[6] S. Subramaniam, K. B. Jayanthi, C. Rajasekaran, and R. Kuchelar, "Deep learning architectures for medical image segmentation," in Proceedings of the 2020 IEEE 33rd International Symposium on Computer-Based Medical Systems (CBMS), pp. 579-584, Rochester, MN, USA, July 2020.

[7] K. Kim, D. Wu, K. Gong et al., "Penalized PET reconstruction using CNN prior," IEEE Transactions on Medical Imaging, vol. 37, no. 6, 2017.

[8] G. Li, L. Bai, C. Zhu, E. Wu, and R. Ma, "A novel method of synthetic CT generation from MR images based on convolutional neural networks," in Proceedings of the 2018 11th International Congress on Image and Signal Processing, BioMedical Engineering and Informatics (CISP-BMEI), Beijing, China, October 2018.

[9] W. Li, J. Li, K. V. Sarma et al., "Path R-CNN for prostate cancer diagnosis and gleason grading of histological images," IEEE Transactions on Medical Imaging, vol. 38, no. 4, pp. 945-954, 2019.

[10] L. Chen, P. Bentley, K. Mori, K. Misawa, M. Fujiwara, and D. Rueckert, "DRINet for medical image segmentation," IEEE Transactions on Medical Imaging, vol. 37, no. 11, pp. 24532462, 2018.

[11] R. Gu, G. Wang, T. Song et al., "CA-net: comprehensive attention convolutional neural networks for explainable medical image segmentation," IEEE Transactions on Medical Imaging, vol. 40, no. 2, pp. 699-711, 2021.

[12] Q.-E. Xiao, P.-C. Chung, H.-W. Tsai et al., "Hematoxylin and eosin (H \& E) stained liver portal area segmentation using multi-scale receptive field convolutional neural network," IEEE Journal on Emerging and Selected Topics in Circuits and Systems, vol. 9, no. 4, pp. 623-634, 2019.

[13] Y. Yu, J. Wang, C. W. Ng et al., "Deep learning enables automated scoring of liver fibrosis stages," Scientific Reports, vol. 8, no. 1, p. 16016, 2018.

[14] D. S. Reddy, R. Bharath, and P. Rajalakshmi, “A novel computer-aided diagnosis framework using deep learning for classification of fatty liver disease in ultrasound imaging," in Proceedings of the 2018 IEEE 20th International Conference on e-Health Networking, Applications and Services (Healthcom), Ostrava, Czech Republic, September 2018.

[15] A. Krizhevsky, I. Sutskever, and G. E. Hinton, "ImageNet classification with deep convolutional neural networks," Communications of the ACM, vol. 60, no. 6, pp. 84-90, 2017.

[16] C. Szegedy, W. Liu, Y. Jia et al., "Going deeper with convolutions," 2015, https://arxiv.org/abs/1409.4842.

[17] K. Simonyan and A. Zisserman, "Very deep convolutional networks for large-scale image recognition," 2015, http:// arxiv.org/abs/1409.1556.

[18] S.-H. Wang, Y.-D. Lv, Y. Sui, S. Liu, S.-J. Wang, and Y.-D. Zhang, "Alcoholism detection by data augmentation and convolutional neural network with stochastic pooling," Journal of Medical Systems, vol. 42, no. 1, p. 2, 2017.

[19] M. Vinícius dos Santos Ferreira, A. O. d. C. Filho, A. D. d. Sousa, A. C. Silva, and M. Gattass, "Convolutional neural network and texture descriptor-based automatic 
detection and diagnosis of glaucoma," Expert Systems with Applications, vol. 110, pp. 250-263, 2018.

[20] S.-H. Wang, K. Muhammad, J. Hong, A. K. Sangaiah, and Y.-D. Zhang, "Alcoholism identification via convolutional neural network based on parametric ReLU, dropout, and batch normalization," Neural Computing and Applications, vol. 32, no. 3, pp. 665-680, 2018.

[21] H. Khosravi, S. I. Saedi, and M. Rezaei, "Real-time recognition of on-branch olive ripening stages by a deep convolutional neural network," Scientia Horticulturae, vol. 287, p. 10, 2021.

[22] M. Teti, W. E. Hahn, S. Martin, C. Teti, and E. Barenholtz, "A controlled investigation of behaviorally-cloned deep neural network behaviors in an autonomous steering task," Robotics and Autonomous Systems, vol. 142, p. 18, 2021. 